\title{
GRAVITATIONAL LENSING OF QUASAR 0957+561 AND THE DETERMINATION OF $\mathrm{H}_{0}$
}

\author{
GEORGE RHEE \\ Department of Physics, University of Nevada, Las Vegas \\ GARY BERNSTEIN \\ Department of Astronomy, University of Michigan \\ AND \\ TONY TYSON AND PHIL FISCHER \\ ATET Bell Labs
}

\section{Introduction}

The double quasar $0957+561$ was the first discovered instance of multiple imaging via gravitational lensing. The galaxy cluster is an important deflector as well as the first ranked galaxy. This has so far precluded construction of a unique model of the lens, reducing the accuracy of the derived $\mathrm{H}_{0}$ value. We have obtained deep images of the system at CFHT. The cluster is sufficiently massive to cause distortions on distant background galaxy images. We have used a mass map derived from lensing distortions to improve the accuracy of the cluster center location and place new limits on $\mathrm{H}_{0}$.

\section{The Time Delay}

Vanderriest et al. (1989) obtain a value of $415 \pm 20$ days based on optical data. Schild (1991) obtains a value of $404 \pm 10$ days also based on optical monitoring. From radio monitoring studies Lehar et al. (1991) conclude that the time delay is $513 \pm 40$ days.

\section{The Mass Distribution}

The galaxy principally responsible for the lensing (G1) has a redshift of 0.36. Bernstein et al. (1993) find that the surface brightness profile is well 
fit by a power law with index $n=-1.94 \pm 0.06$. The position angle of the galaxy may twist slightly about a mean value of $55^{\circ}$. From spectroscopic observations Rhee (1991) derives a velocity dispersion $300 \pm 50 \mathrm{~km} \mathrm{~s}^{-1}$ for G1.

The parameters for the galaxy model are: the velocity dispersion, the ellipticity, the galaxy position angle and the power law index. The cluster is modeled as a pseudo-isothermal sphere. The cluster maps show that the cluster potential is not perfectly smooth. This can be taken into account by adding a shear component to the two components listed above. The shear component has two parameters the magnitude of the shear and its position angle. The effect of the shear may be taken as some indication of the changes in the models that would result from the addition of lumps to the mass distribution.

The observations were made with FOCAM and the SAIC CCD at CFHT in January 1994. We obtained a 3 hour integration in B band and a 4 hour integration in I-band. By inverting the estimated shear field inferred from the observed shapes and orientations of background galaxies we have obtained a map of the surface mass density of the matter lensing Q0957+561.

\section{Conclusion}

From the map of the mass distribution we can locate the center of the cluster relative to the lensing galaxy. We measure the cluster center position to be at a distance $\left(\mathrm{r}_{c}\right)$ of 9 arcseconds from the lensing galaxy in the direction $\theta_{c}=193^{\circ}$. By limiting the range in values that these two parameters can have we eliminate all models except those having $\mathrm{H}_{0}$ less than $70 \mathrm{~km} \mathrm{~s}^{-1}$ $\mathrm{Mpc}^{-1}$.

\section{Acknowledgements}

George Rhee acknowledges travel support from the UNLV Physics department Bigelow fund and accommodation support from an IAU grant.

\section{References}

Bernstein, G.M., Tyson, J.A., \& Kochanek, C.S. 1993, AJ, 105, 816

Lehar, J., Hewitt, J.N., \& Roberts, D.H., 1989, in Gravitational Lenses, ed. J.M. Moran, J.N. Hewitt, \& K.Y. Lo (Berlin: Springer) 84

Rhee, G. 1991, Nature, 350, 211

Schild, R., AJ, 1990, 100, 1771

Vanderriest, C., Schneider, J., Herpe, G., Chevreton, M., Moles, M., \& Wlerick, G. 1989, A\&A, 215, 1 